Silvia Hansen-Schirra/Katharina Oster/Jean Nitzke/ Anne-Kathrin Gros (Mainz)

\title{
Die Rolle der Entropie im Übersetzungsprozess
}

\begin{abstract}
Die Sprachverarbeitung beim Übersetzen unterliegt zwei gegenläufigen Forderungen: der ausgangstextbasierten Äquivalenzforderung und der funktionalistischen Zielpublikumsorientierung. So können Übersetzungen mehr oder weniger wie eine Kopie des Ausgangstextes in einer anderen Sprache wirken, je nachdem wie wörtlich oder frei übersetzt wurde. Dieses Entscheidungskontinuum lässt sich mit dem Entropiebegriff operationalisieren. Je höher die Entropie, desto mehr Übersetzungsvarianten gibt es für einen ausgangssprachlichen Ausdruck. Welche Rolle hierbei das mentale Lexikon spielt und inwiefern die Entropie die kognitiven Prozesse beim Übersetzen beeinflusst, kann durch experimentelle Forschung untersucht werden.

In einer ersten Studie haben wir den Einfluss des mentalen Lexikons auf die Übersetzungsentropie und dessen Entwicklungspotenzial bei Studierenden am Beispiel von Kognaten untersucht. Die zweite Studie belegt den Zusammenhang zwischen Entropie und der kognitiven Belastung am Bespiel verschiedener Wortarten. Durch die Datentriangulation von produkt- und prozessbasierten Ergebnissen lassen sich spezifische Verwendungsmuster ableiten.
\end{abstract}

\section{Entscheidungskontinuum beim Übersetzen}

Beim Übersetzen liegt ein generelles Entscheidungskontinuum zwischen wörtlichen und freien Übersetzungsvarianten vor (Catford 1965; Wilss 1996). Hierbei sind die wörtlichen Varianten eher ausgangssprachenorientiert, die freien Varianten eher zielsprachenorientiert. Diese Ausprägungen wurden korpuslinguistisch auch als Shining-through (Teich 2003) vs. Normalisierung (Baker 1996) getestet. Halverson (2003) verfolgt bei der Ursachenbestimmung dieser Phänomene einen kognitiven Ansatz. Ihre Literal Translation Hypothesis besagt Folgendes: Bei der Wahl zwischen einer wörtlichen Lösung, deren Verarbeitung nicht viel kognitiven Aufwand benötigt, und einer Lösung, die mehr Verarbeitung benötigt, entscheidet sich der Übersetzer für die wörtliche, da er so Verarbeitungszeit und kognitive Leistung einspart (vgl. ebd., S. 223). Halversons Erklärungsansatz fußt 
auf de Groots Distributed Conceptual Feature Model (de Groot 1992, S. 393), nach dem Übersetzungsäquivalente über gemeinsame semantische Merkmale auf der konzeptuellen Ebene des bilingualen Lexikons verfügen. Wörter, die viele gemeinsame semantische Merkmale aufweisen, können leichter übersetzt werden. Halverson argumentiert, dass semantische und phonologische Informationen grammatikalischer Einheiten in komplexen Netzwerken verbunden sind. Durch regelmäßige Nutzung einer dieser Verbindungen werden sie verstärkt und es bildet sich eine prototypische Verknüpfung, die bei mentalen Auswahlprozessen bevorzugt wird. Der Prototyp wird „angezogen“. Halverson (2003) nennt diesen Effekt Gravitational Pull. In Bezug auf die Literal Translation Hypothesis wird die bevorzugte Auswahl einer wörtlichen Übersetzung mittels Halversons Gravitational Pull Hypothesis dargelegt: „highly salient structures will exert a gravitational pull, resulting in an overrepresentation in translation of the specific TL lexical and grammatical structures“ (ebd., S. 218).

Englund Dimitrova (2005) und Chesterman (2011) ziehen vor allem psycholinguistische Erklärungsansätze für die Literal Translation Hypothesis in Erwägung. Sie bezeichnen die Neigung zur Literal Translation und auch deren Revidierung als Übersetzungsstrategie. Krings (1986) definiert Übersetzungsstrategien als „potentiell bewusste Pläne eines Übersetzers zur Lösung konkreter Übersetzungsprobleme im Rahmen einer konkreten Übersetzungsaufgabe“ (ebd., S. 175). Das Wort potentiell soll an dieser Stelle hervorgehoben werden, da Strategien auch unbewusst und automatisiert greifen können. Englund Dimitrova sieht einen potentiellen Zusammenhang zwischen translatorischer Kompetenz und dem Automatisierungsgrad übersetzerischer Strategien:

The professional translator can be assumed to have worked out, or perhaps explicitly learnt, certain (types of) solutions to recurring textual phenomena in his/her particular language combination. In the translation process, such ready-made solutions can be assumed to be automated to a certain extent. (Englund Dimitrova 2005, S. 59)

Hier wird vermutet, dass bei professionellen Übersetzern auch erlernte Übersetzungsstrategien in einem hohen Maße verinnerlicht sind, sodass man von automatisierten Strategien sprechen kann. Strittig bleibt jedoch, ob erlernte Strategien derartig in das übersetzerische Verhalten eingehen können, als dass von einer automatisierten mentalen Reaktion gesprochen werden kann.

Tirkkonen-Condit (2004) und Halverson (2015) (ebenso Schaeffer/Carl 2013) argumentieren für eine kognitive Grundlage der Literal Translation Hypothesis und sehen die Ursprünge des Phänomens in der Struktur des bilingualen Lexikons. Sollte diese Interpretation und damit der Ursprung von Literal Translations in mentalen Prozessen, v.a. im sogenannten Priming bewiesen werden 
können, kann angenommen werden, dass Literal Translations automatisch produziert werden und nicht steuerbar sind. Somit qualifiziert sich das Phänomen als Übersetzungsuniversalie.

Um das Entscheidungskontinuum zwischen wörtlicher vs. freier Übersetzung zu operationalisieren, haben Schaeffer et al. (2016), wie im Folgenden ausgeführt, den Entropiebegriff für die Übersetzungswissenschaft adaptiert. Die Entropie ist ein Konzept aus der Thermodynamik und wird häufig als Maß der Unordnung beschrieben. Claude E. Shannon (1948), Begründer der Informationstheorie, nutzte den Entropiebegriff zur theoretischen Betrachtung von Kommunikation. In diesem Zusammenhang verknüpft Entropie das Konzept des Informationsgehalts I einer Nachricht mit der Auftrittswahrscheinlichkeit dieser Nachricht. Der Informationsgehalt $I$ einer Nachricht in Abhängigkeit ihrer Auftrittswahrscheinlichkeit $p$ errechnet sich nach Shannon (1948, S. 48) wie folgt:

$$
I(p)=-\log 2(p)
$$

Dies bedeutet, dass eine Nachricht mit einer geringen Auftrittswahrscheinlichkeit einen höheren Informationsgehalt besitzt als eine Nachricht, die oft auftritt. Diese Abhängigkeit des Informationsgehaltes von der Auftrittswahrscheinlichkeit wird von Shannon als logarithmisch angenommen. Um auszudrücken, wie unsicher das Auftreten einer Information ist, wird aus dem Informationsgehalt der Auftrittswahrscheinlichkeit die Entropie $H$ über die folgende Formel berechnet:

$$
H=\sum \text { pini }=1 I(p i)=- \text { Spini }=1 \log 2(p i)
$$

Der Entropiebegriff wurde in der Translationswissenschaft als Maß der Unsicherheit (Degree of Uncertainty; Schaeffer et al. 2016, S. 191) operationalisiert und beschreibt gleichzeitig die Anzahl von Übersetzungsalternativen einer lexikalischen Einheit und die Wahrscheinlichkeit ihres Vorkommens (bzw. dass sie als Alternative ausgewählt werden) in einem Korpus.

Wird angenommen, dass alle Übersetzungsalternativen einer lexikalischen Einheit gleich wahrscheinlich sind, so fällt der Entropiewert hoch aus. Existieren zwar viele Übersetzungsalternativen, jedoch kommen einige nur sehr selten vor und eine sehr häufig, ist der Entropiewert niedrig. In der Praxis bedeutet das, wenn ein Wort $n$ mal übersetzt und von $m$ Übersetzern immer gleich übersetzt wurde, weist dieses Wort in Bezug auf den Korpus der $m$ Übersetzer eine Entropie von 0 auf und wird laut Schaeffer et al. (2016) als prototypische Default Translation angesehen. Welche Rolle das mentale Lexikon auf die Übersetzungsentropie hat und diese wiederum auf die kognitive Verarbeitung beim Übersetzen, wird im Folgenden empirisch untersucht. 


\section{2 Übersetzungsentropie in Abhängigkeit des mentalen Lexikons}

Welchen Einfluss hat das mentale Lexikon auf die Entropie? Eine Methode, das mentale Lexikon zu untersuchen, sind Word Translation Tests. Bei diesen Experimenten werden einzelne Wörter von Probanden so schnell wie möglich mündlich in eine andere Sprache übersetzt. Durch eine experimentelle Manipulation der Stimuli können so die Reaktionszeiten auf verschiedene linguistische Parameter untersucht werden. Je kürzer dabei die Reaktionszeiten sind, desto schneller ist die mentale Verarbeitung. Bei Word Translation Tests bedeutet dies, dass die Übersetzung zum Beispiel von Wörtern bestimmter Wortklassen einfacher ist.

Ein bekannter Effekt, der bei diesen Experimenten untersucht werden kann, ist der Cognate Facilitation Effect. Es wurde festgestellt, dass Kognaten (Übersetzungsäquivalente mit einer ähnlichen Form, z.B. system (Englisch) und System (Deutsch)) schneller übersetzt werden als Nicht-Kognaten (Übersetzungsäquivalente mit unterschiedlicher Form, z.B. health (Englisch) und Gesundheit (Deutsch)) (Christoffels/de Groot/Kroll 2006). Die Differenz in der Reaktionszeit ist der Cognate Facilitation Effect. Dieses Ergebnis wird damit erklärt, dass Kognaten auf Grund ihrer ähnlichen Form im mentalen Lexikon eng miteinander verknüpft sind. Während der Rezeption werden daher Kognaten voraktiviert und können während der Übersetzung somit leichter produziert werden als Nicht-Kognaten.

Es wäre nun möglich, dass dieser Erleichterungseffekt nicht nur mit der Reaktionszeit, sondern auch mit der Art des Outputs in Verbindung gebracht werden kann. Dies könnte dann ein erster Hinweis darauf sein, dass die Struktur des mentalen Lexikons auch eine Rolle für die Entropie spielt. Um dies zu untersuchen, haben wir einen Word Translation Test durchgeführt und neben den Reaktionszeiten auch gemessen, wie viele ausgangssprachliche Kognaten mit zielsprachlichen Kognaten bzw. Nicht-Kognaten übersetzt wurden. Diese Messmethode steht in direktem Zusammenhang zu den Parametern des Word Translation Tests und ist relativ einfach zu messen. Sie stellt zudem ein grobes Maß für Entropie dar. Für weitere Auswertungen ist natürlich auch eine genauere Analyse der Entropie denkbar.

An dem Word Translation Test nahmen 20 Übersetzungsstudierende teil. Als Stimuli dienten jeweils 44 abstrakte Kognaten und Nicht-Kognaten, die sich nicht hinsichtlich der Wortlänge in Buchstaben, der Frequenz (BNC) und der möglichen Übersetzungen (www.linguee.de) unterscheiden (siehe Tab. 1). Die Probanden erhielten den Auftrag, die Wörter so schnell und so präzise wie möglich aus dem Englischen ins Deutsche zu übersetzen. 
Tab. 1: Beispielstimuli für den Word Translation Test

\begin{tabular}{ll}
\hline Kognaten & Nicht-Kognaten \\
\hline activity & complaint \\
comment & decrease \\
factor & amount \\
project & growth \\
method & fear \\
\hline
\end{tabular}

Das Experiment bestand aus einem Probeblock und 10 experimentellen Blöcken. Jeder Block beinhaltete vier Kognaten und vier Nicht-Kognaten, die jeweils viermal wiederholt wurden. Die Anzeige der Stimuli erfolgte dabei in einer pseudorandomisierten Reihenfolge. Insgesamt bestand das Experiment somit aus 32 Stimuli im Probeblock und 320 Stimuli im experimentellen Teil.

Vor jedem Stimulus erschien ein Fixationskreuz für 500-800 ms, gefolgt von einem schwarzen Bildschirm, der auch 500 ms eingeblendet wurde. Der Stimulus erschien für $500 \mathrm{~ms}$ auf dem Bildschirm gefolgt von einem schwarzen Bildschirm für 2000 ms. Die nächsten Fixationskreuze und Stimuli erschienen automatisch, ohne dass der Proband weiterklicken musste. Nach jedem Block konnten die Probanden eine Pause machen und selbst entscheiden, wann sie weitermachen wollten.

Die Präsentation der Stimuli erfolgte mit der Software Psychopy, der Sprachoutput der Probanden wurde mit Audacity und die Reaktionszeiten mit Hilfe eines Voice Keys (Cedrus Stim Tracker) aufgezeichnet.

Die mit Hilfe des Voice Keys aufgezeichneten Reaktionszeiten wurden für jeden Probanden für Kognaten und Nicht-Kognaten gemittelt. Dabei fanden nur korrekte Antworten Berücksichtigung. Zudem entfernten wir Antworten, vor denen ein Störgeräusch in den Audioaufzeichnungen zu hören war, wie zum Beispiel ein Husten oder ein ähm, da dies den Voice Key fälschlicherweise zu früh hätte auslösen können.

Um den Cognate Facilitation Effect zu berechnen, wurde dann die Reaktionszeit für Kognaten von der Reaktionszeit für Nicht-Kognaten abgezogen:

$$
\text { RT_Non_Cognates }- \text { RT_Cognates }=\text { CFE }
$$

Da wir untersuchen wollten, ob die Art, wie Wörter übersetzt werden, mit dem Cognate Facilitation Effect korreliert, werteten wir außerdem aus, bei wie vielen Kognaten die Übersetzung durch die entsprechenden Kognaten bzw. durch NichtKognaten erfolgte. Beispiel: project wurde nicht mit Projekt, sondern mit Vorhaben übersetzt. 
In einem letzten Schritt haben wir dann den Zusammenhang zwischen der Anzahl der übersetzten Nicht-Kognaten und dem Cognate Facilitation Effect berechnet (siehe Abb. 1). Dabei stellte sich heraus, dass der Cognate Facilitation Effect stark mit der Anzahl der Nicht-Kognaten korreliert: r(18)=-.67, p=.0012.

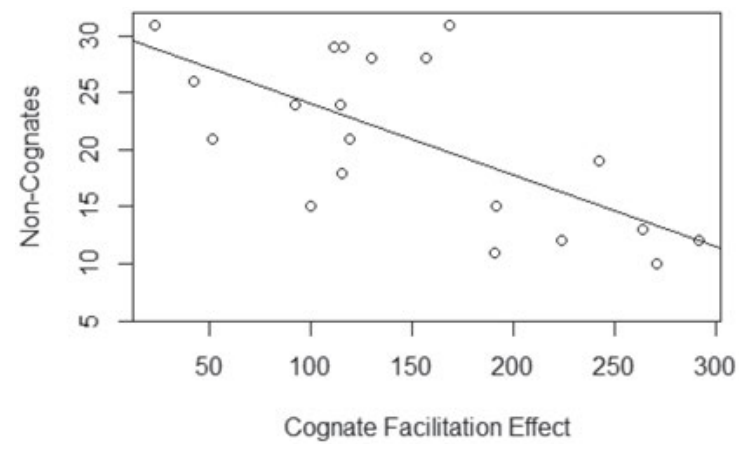

Abb. 1: Verhältnis von Cognate Facilitation Effect zu übersetzten Nicht-Kognaten

Die Ergebnisse des Word Translation Tests zeigen, dass es einen Zusammenhang zwischen den für eine Übersetzung gewählten Wörtern und dem Cognate Facilitation Effect und damit dem mentalen Lexikon gibt. Diese Studie wurde nur mit 20 Probanden durchgeführt und andere Variablen außer dem Cognate Facilitation Effect und der Anzahl der übersetzten Nicht-Kognaten wurden nicht berücksichtigt. Eine Ausweitung der Studie ist daher geplant (siehe Oster i.Vorb.). Diese ersten Ergebnisse weisen allerdings darauf hin, dass es nicht nur eine Verbindung zwischen der Struktur des mentalen Lexikons und Reaktionszeiten gibt, sondern dass auch eine Verbindung zwischen dem mentalen Lexikon und der Art des Outputs gibt. Es könnte sich daher lohnen, Experimente zum mentalen Lexikon in Betracht zu ziehen, wenn unterschiedliche Entropiewerte zwischen verschiedenen Populationen beim Übersetzen beobachtet werden.

Um zu überprüfen, inwiefern sich das mentale Lexikon beim Übersetzen mit steigender Kompetenz entwickelt, haben wir ein weiteres Experiment durchgeführt, in dem 43 Übersetzungsstudierende (alle deutsche Muttersprachler) des Fachbereichs Translations-, Sprach- und Kulturwissenschaft (FTSK) in Germersheim aufgefordert wurden, einen Text aus dem Englischen zu übersetzen. Der Text umfasste 187 Wörter und enthielt 49 englisch-deutsche Kognaten, die wir als Stimuli für die Übersetzungsentscheidungen untersuchten. Für die Auswertung zählten wir, wie oft die Kognaten in der Übersetzung mit Kognaten bzw. mit NichtKognaten übersetzt wurden und korrelierten den Befund mit der Semesterzahl, die die Probanden in den Metadatenfragebögen angaben. 
Die Ergebnisse in Abbildung 2 zeigen, dass die Anzahl der Kognaten-Übersetzung negativ mit der Semesterzahl korreliert. Das heißt, je fortgeschrittener die Probanden im Studienverlauf sind, desto häufiger übersetzen sie die KognatenStimuli mit Nicht-Kognaten. Dies lässt vermuten, dass sich ein Mechanismus bei Übersetzungsstudierenden entwickelt, der die Auswahlentscheidung für NichtKognaten präferiert. Dies könnte durch Veränderungen im mentalen Lexikon bedingt sein, denn es wurde bereits gezeigt, dass auch im Erwachsenenalter neue Wörter gelernt werden können und Verbindungen in der netzwerkähnlichen Struktur des mentalen Lexikons verstärkt oder geschwächt werden können (Aitchison 2012). Der Grund könnte aber auch auf einen ausgeprägten Monitoring-Mechanismus zurückzuführen sein (Hansen-Schirra/Nitzke/Oster i.Dr.; Oster i.Vorb.; siehe auch Levelt 1999). Allerdings haben mehrere Studien festgestellt, dass sich das Monitoring nach der Kindheit nicht mehr entwickelt (z.B. Wiersema/van der Meere/Roeyers et al. 2007), dessen Ausprägung und Intensität hängt jedoch von den vorhandenen mentalen Ressourcen ab: z.B. Motivation (Ganushchak/ Schiller 2008) oder Zeitdruck (Ganushchak/Schiller 2006).

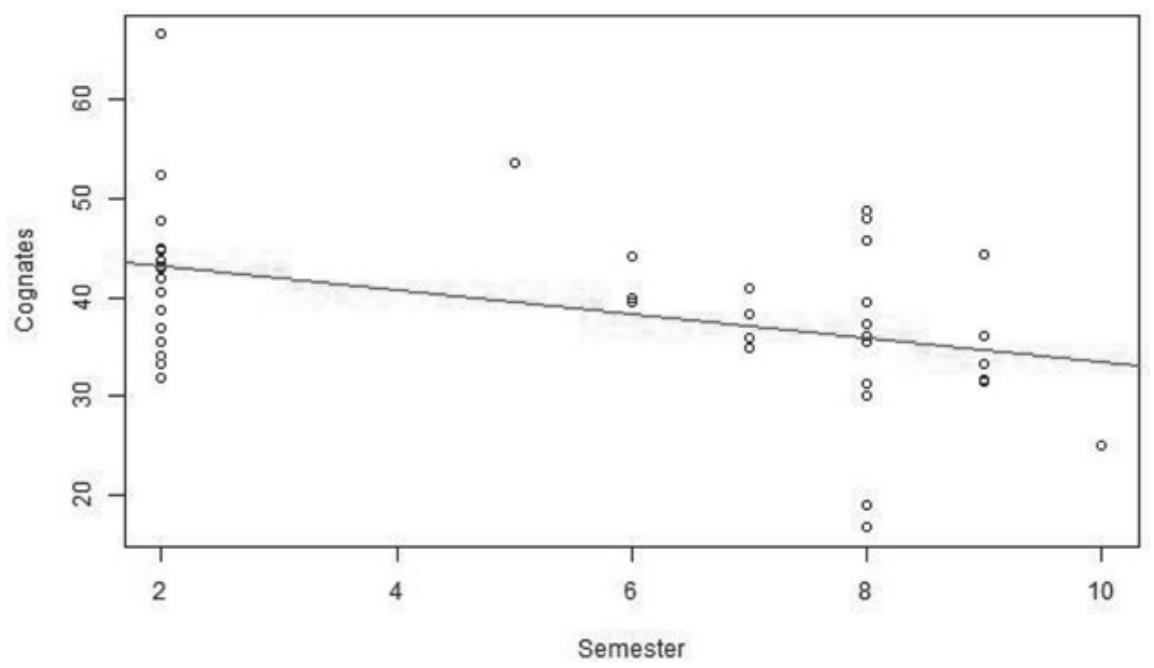

Abb. 2: Negative Korrelation zwischen Kognaten-Übersetzung und Semesterzahl $(r(41)=-.42, p=.005)$

Unsere Hypothese lautet demnach, dass sich das mentale Lexikon weiter entwickelt. Die Verbindungen zwischen Nicht-Kognaten werden stärker, da Kognaten ständig durch den Monitoring-Prozess gefiltert werden. Wir vermuten, dass sich der Kontrollmechanismus selbst nicht weiterentwickelt. Aber da der Über- 
setzer weniger kognitive Ressourcen benötigt, um Nicht-Kognaten zu aktivieren (die Ko-Aktivierungsschwelle wird im Laufe der Zeit gesenkt), stehen mehr kognitive Ressourcen für die Monitoring-Prozesse zur Verfügung. Es muss jedoch bedacht werden, dass die Ergebnisse nicht nur auf die Übersetzerausbildung zurückzuführen sind, sondern auch auf eine erhöhte bilinguale Kompetenz. Daher ist zu vermuten, dass diese Expertise mit der Entwicklung des mentalen Lexikons einhergeht.

\section{Kognitive Prozesse in Abhängigkeit der Übersetzungsentropie}

Wie oben bereits beschrieben, gibt die Entropie in der Translationswissenschaft die Unsicherheit der Übersetzung wieder, d.h. je höher ein Entropiewert ist, umso mehr Übersetzungsmöglichkeiten gibt es für die ausgangssprachliche Einheit. Dabei spielt zum einen die Anzahl der Übersetzungsmöglichkeiten eine Rolle, zum anderen aber auch die Wahrscheinlichkeiten, dass diese Möglichkeiten gewählt werden. Wenn in einem parallelen Korpus eine Texteinheit sehr selten mit einer Variante mit einer anderen hingegen sehr häufig übersetzt wurde, ist der Entropiewert für die seltene Einheit kleiner, als wenn beide Übersetzungsmöglichkeiten ungefähr gleich häufig gewählt wurden (vgl. Bangalore et al. 2016, S. 213-214).

Um den Effekt von Entropie auf die kognitiven Übersetzungsprozesse in verschiedenen Übersetzungs- und Bearbeitungsmodi messbar zu machen, wurden in einer Experimentreihe 24 Übersetzer (12 professionelle Übersetzer, 12 Studierende der Translation) gebeten, sechs Texte vom Englischen ins Deutsche zu übersetzen, zu post-editieren und monolingual zu post-editieren. ${ }^{1}$ Die Texte waren jeweils 110-160 Wörter lang und beschäftigten sich mit eher allgemeinsprachlichen Themen. Außerdem wurden von jedem Probanden Keylogging- und Eyetracking-Daten mithilfe der Software Translog II (Carl 2012) und einem Tobii TX300 aufgezeichnet. Die Daten sind frei zugänglich in der CRITT TPR-DB-Datenbank $^{2}$ (Carl/Bangalore/Schaeffer (Hg.) 2016), initiiert von der Copenhagen Busi-

1 Post-Editing bezeichnet die Nachbearbeitung maschineller Übersetzung durch einen Übersetzer. Der Begriff bezieht sich in der Regel auf das bilinguale Post-Editing, bei dem dem Übersetzer auch der Ausgangstext zur Verfügung steht. Beim monolingualen Post-Editing steht dem Übersetzer nur die maschinelle Übersetzung zur Verfügung.

$2 \mathrm{https} / /$ sites.google.com/site/centretranslationinnovation/tpr-db. 
ness School. Die Datenbank besteht aus Translationsprozessdaten, aufgenommen in verschiedenen Experimentszenarien und in unterschiedlichsten Sprachkombinationen. Die vorliegende Datenreihe wurde beispielsweise nicht nur vom Englischen ins Deutsche, sondern auch ins Japanische, Spanische, Chinesische, Dänische und in Hindi über die verschiedenen Modi transferiert. Für die Auswertung wurde Version 2.310 der Datenbank verwendet.

Die Entropiewerte wurden automatisch probanden- und aufgabenübergreifend berechnet, d.h. dass alle Translationsvarianten in die Werte einfließen, egal ob ohne maschinelle Vorarbeit übersetzt oder (monolingual) post-editiert wurde. Im Folgenden werden allerdings nur die Keylogging- und Eyetracking-Daten der Humanübersetzungen ausgewertet.

$\mathrm{Zu}$ untersuchen ist die Hypothese, ob sich die Entropie der einzelnen Wörter auf die kognitive Verarbeitung (gemessen an Keylogging- und Eyetracking-Daten, d.h. kognitiver Aufwand für die Schreib- und Leseprozesse beim Übersetzen) auswirkt (Nitzke i.Vorb.). Vermutet wird, dass die Entropiewerte einen statistisch signifikanten Einfluss auf die Prozessdaten haben - je höher die Entropie, desto höher der kognitive Aufwand $\left(\mathrm{H}_{1}\right)$. Weiterhin gehen wir davon aus, dass einige Wortarten bei hoher Entropie mehr kognitiven Aufwand als andere veranschlagen $\left(\mathrm{H}_{2}\right)$.

Für die Auswertung wurde ein lineares gemischtes Modell in R erstellt (R Core Team 2000), um herauszufinden, welchen Einfluss Wortentropie (HTra) auf die Prozessdaten hat. Als weitere Einflussfaktoren wurden Status und Erfahrung der Probanden mit ins Modell aufgenommen. Der Status der Probanden ist in Studierende und professionelle Übersetzer aufgeteilt. Da die Gruppen in sich selbst sehr heterogen sind, wurde ein Erfahrungsfaktor eingeführt, um besser abzubilden, wie viel Erfahrung die Probanden haben. In diesem Faktor werden Studienjahre einfach, Jahre professioneller Übersetzererfahrung doppelt gezählt (ausführliche Beschreibung in Nitzke 2016). Die einzelnen Probanden sowie die verschiedenen Texte wurden als Zufallseffekte aufgenommen. Das statistische Modell wurde somit mit dem folgenden Aufruf berechnet:

$$
\begin{aligned}
& \text { Imer }(\text { Prozessparameter } \sim \\
& \text { HTra }+ \text { Status }+ \text { Exp }+(1 / \text { Part })+(1 / \text { Text }), \text { dataset })
\end{aligned}
$$

Wenn kein signifikanter Einfluss von Status und Erfahrung (Exp) nachgewiesen werden konnte, wurden die entsprechenden Faktoren aus dem Modell ausgeschlossen. Die getesteten Prozessparameter (vgl. Carl/Bangalore/Schaeffer (Hg.) et al. 2016) umfassen die Produktionsdauer (Dur), die Gesamtdauer der Fixationen im Ausgangstext (TrtS), die Gesamtzahl der Fixationen im Ausgangstext (FixS), die Gesamtdauer der Fixationen im Zieltext (TrtT) und die Gesamtzahl der Fixationen im Zieltext (FixT). Der Einfluss der Wortentropie wurde sowohl auf die Ge- 
samtdaten als auch auf die einzelnen Wortarten getestet. ${ }^{3}$ Aufgrund geringen Vorkommens in den Texten, wurden Adjektive, Adverbien und deren Komparationen, sowie wh-Wörter (Determinativ, Pronomen und Adverbien) zusammengefasst. Des Weiteren wurden zusätzlich zu den Unterkategorien inkludierende Kategorien für Nomen und Verben erstellt.

Wenn alle Datenpunkte in Betracht gezogen werden, sind die Ergebnisse signifikant für Gesamtdauer $(\mathrm{t}=3.56, \mathrm{p}=0.0004)$ und Anzahl der Fixationen auf den Zieltext $(t=3.17, p=0.0015)$, die Entropie eines Wortes beeinflusst also das Blickverhalten auf den Zieltext. Jedoch kann dieses Ergebnis nicht auf alle Wortarten übertragen werden. Die Ergebnisse, die im Folgenden diskutiert werden, beziehen sich auf alle sechs Texte, wobei jeder dieser Texte sieben bis acht ${ }^{4} \mathrm{Mal}$ übersetzt wurde. Es muss daher berücksichtigt werden, dass die teilweise geringen Vorkommen in dem recht kleinen Textkorpus dafür gesorgt haben können, dass die Entropiewerte keinen signifikanten Einfluss hatten, da die Stichproben zu klein sind. In den wenigen Fällen, in denen sowohl Entropie als auch Status/ Erfahrung der Probanden einen signifikanten Effekt hatten, wurden die Variablen auf Kollinearität überprüft. ${ }^{5}$ All diese Tests verliefen negativ, weshalb kein Faktor aus dem Modell entfernt werden musste.

Die Produktionsdauer (Dur) scheint allein bei Präpositionen und Verben durch die Wortentropie beeinflusst zu sein. Des Weiteren hat eine große Variation in der Übersetzung von Präpositionen auch einen Einfluss auf das Leseverhalten im Zieltext. Während Entropie bei den einzelnen Unterkategorien der Verben, wenn überhaupt, einen Einfluss auf die Blickbewegung im Zieltext hat, besteht für die Gesamtgruppe Verben ein Zusammenhang zwischen Entropie und Blickbewegung im Ausgangstext. Auch bei den Nomen beeinflusst die Entropie das Leseverhalten im Ausgangstext und die Anzahl der Fixationen im Zieltext. Überraschenderweise zeigen Kardinalzahlen sowohl im Leseverhalten im Ausgangs- als auch Zieltext signifikante Zusammenhänge zur Entropie. Dies könnte daran liegen, dass man im Allgemeinen nicht viel Variation bei der Übersetzung von Zahlen erwartet und an den Stellen, an denen verschiedene Übersetzungsmöglichkeiten abgewogen werden müssen, der kognitive Aufwand signifikant höher ist (da auch davon auszugehen ist, dass die Übersetzung von Zahlen i.d.R. keine großen Schwierigkeiten verursacht, wenn bspw. keine Einheiten umgerechnet werden müssen).

3 Das Part-of-Speech-Tagging ist bereits in der Datenbank enthalten und richtet sich nach der Kategorien der Penn Treebank (vgl. Marcus/Marcinkiewicz/Santorini 1993).

4 Aus technischen Gründen wurden einige Sitzungen fehlerhaft oder gar nicht aufgezeichnet.

5 Das entsprechende Skript wurde von Austin F. Frank entwickelt und ist verfügbar unter: https://raw.github.com/aufrank/R-hacks/master/mer-utils.R. 
Des Weiteren werden die Schreib- und Blickdaten der Adjektive gar nicht von Entropie beeinflusst, was wiederum daran liegen könnte, dass sich deren Bedeutung aus dem Kontext oft unproblematisch erschließen lässt oder sie feste Bestandteile in Kollokationen darstellen.

Bei den meisten Ergebnissen ist der t-Wert positiv, d.h. die Ergebnisse sind positiv gerichtet. Wenn die Entropie also hoch ist, sind auch die Fixationsdauer sowie die Anzahl der Tastaturbewegungen erhöht. Jedoch gibt es auch einige wenige Fälle, bei denen die Ergebnisse negativ gerichtet sind, die Fixationsdauer und die Anzahl an Tastaturbewegungen sinken, wenn die Entropie steigt, z.B. bei den Adverbien, deren Lesezeiten im Ausgangstext signifikant von der Entropie beeinflusst werden. Ein Grund dafür könnte sein, dass englische Adverbien mit deutschen Adverbialphrasen übersetzt werden, was eine standardisierte Strategie und daher kognitiv wenig aufwändig ist. Dieses Phänomen erfordert allerdings noch weitere Forschung.

Die Hypothesen können zusammenfassend nur teilweise bestätigt werden. Einerseits hat sich gezeigt, dass Entropie oft einen signifikanten Einfluss auf die kognitiven Übersetzungsprozesse hat, aber nicht auf jeden Parameter. Auch hat sich gezeigt, dass einige Wortarten, wie z.B. die Kardinalzahlen, sensibler auf Entropie reagieren als andere. Adjektive beispielsweise wurden überhaupt nicht signifikant von Entropie beeinflusst, obwohl sie im Vergleich $\mathrm{zu}$ anderen Wortarten relativ häufig vorkommen. Inwiefern dies auf automatisierte Prozesse beim Übersetzen schließen lässt, muss Gegenstand weiterführender Studien sein (siehe z.B. die EEG-Studie von Oster i.Vorb.).

\section{Zusammenfassung und Ausblick}

Das Entscheidungskontinuum zwischen wörtlicher und freier Übersetzung lässt sich empirisch mit dem Entropiebegriff operationalisieren. Unsere Studien zeigen einerseits, welche Rolle das mentale Lexikon bei der Übersetzung von Kognaten und den damit verbundenen Effekt auf die Übersetzungsentropie spielen kann. Andererseits haben wir gezeigt, dass Entropie ihrerseits Auswirkungen auf die kognitiven Prozesse und insbesondere die kognitive Belastung beim Übersetzen haben kann. Letzteres wurde mithilfe von Keylogging- und Eyetracking-Daten operationalisiert.

Als Einschränkung kann aufgeführt werden, dass bei dem Word Translation Test bisher nur mit Kognaten gearbeitet wurde. Dies hatte zwar den Vorteil, dass die Manipulation der Stimuli sehr systematisch erfolgen und ausgewertet werden konnte; der Nachteil besteht jedoch darin, dass man entsprechend nur die Entro- 
piewerte für Kognaten ableiten kann. Diese Studie müsste mit einem breiten Spektrum an lexikalischen Einheiten wiederholt werden. Weiterhin wäre es spannend, ereigniskorrelierte Potentiale über EEG-Untersuchungen abzuleiten, um Primingund Monitoring-Effekte auch physiologisch nachweisen zu können.

Die Triangulation von Entropiewerten und Eyetracking- und Keylogging-Daten aus der CRITT TPR-DB stellt eine innovative Methode dar, die vielversprechende Einblicke in die kognitiven Verarbeitungsmechanismen beim Übersetzen ermöglicht. Wie jedoch genau der Zusammenhang zwischen den Wortarten, der Entropie und der kognitiven Belastung beim Übersetzen zu erklären ist, bleibt auf der Basis dieser Datengrundlage immer noch unklar. Hierfür müssten tiefergehende und stärker kontrollierte Experimente durchgeführt werden, um die Variablen isoliert interpretieren $\mathrm{zu}$ können.

\section{Literatur}

Aitchison, Jean (2012): Words in the mind. An introduction to the mental lexicon. 4. Aufl. Oxford.

Baker, Mona (1996): Corpus-based translation studies. The challenges that lie ahead. In: Somers, Harold (Hg.): Terminology, LSP and translation. Studies in language engineering in honour of Juan C. Sager. (= Benjamin Translation Library 18). Amsterdam/Philadelphia, S. $175-186$.

Bangalore, Srinivas et al. (2016): Syntactic variance and priming effects in translation. In: Carl/ Bangalore/Schaeffer (Hg.), S. 211-238.

Carl, Michael (2012): Translog-II: A program for recording user activity data for empirical reading and writing research. In: The Proceedings of the Eighth International Conference on Language Resources and Evaluation (LREC). Istanbul, S. 4108-4112.

Carl, Michael/Bangalore, Srinivas/Schaeffer, Moritz (2016): The CRITT Translation Process Research Database. Exploring the CRITT TPR-DB. In: Carl/Bangalore/Schaeffer (Hg.), S. 13-54.

Carl, Michael/Bangalore, Srinivas/Schaeffer, Moritz (Hg.) (2016): New directions in empirical translation process research. Exploring the CRITT TPR-DB. Cham u.a.

Catford, John C. (1965): A linguistic theory of translation. An essay in applied linguistics. (= Language and Language Learning 8). London.

Chesterman, Andrew (2011): Reflections on the literal translation hypothesis. In: Alvstad, Cecilia/Hild, Adelina/Tiselius, Elisabet (Hg.): Methods and strategies of process research: Integrative approaches in translation studies. (= Benjamins Translation Library 94). Amsterdam u.a., S. 23-35.

Christoffels, Ingrid K./de Groot, Annette M.B./Kroll, Judith F. (2006): Memory and language skills in simultaneous interpreters: The role of expertise and language proficiency. In: Journal of Memory and Language 54, 3, S. 324-345.

de Groot, Annette M. (1992): Bilingual lexical representation. A closer look at conceptual representations. In: Advances in Psychology 94, S. 389-412. 
Englund Dimitrova, Birgitta (2005): Expertise and explicitation in the translation process. (= Benjamins Translation Library 64). Amsterdam/Philadelphia.

Ganushchak, Lesya Y./Schiller, Niels O. (2006): Effects of time pressure on verbal selfmonitoring. An ERP study. In: Brain Research 1125, 1, S. 104-115.

Ganushchak, Lesya Y./Schiller, Niels 0. (2008): Motivation and semantic context affect brain error-monitoring activity: An event-related brain potentials study. In: Neurolmage 39, 1, S. 395-405.

Hansen-Schirra, Silvia/Nitzke, Jean/Oster, Katharina (i.Dr.): Predicting the use of cognates in translation. In: Hansen-Schirra, Silvia et al. (Hg.): Empirical modelling of translation and interpreting. Berlin.

Halverson, Sandra L. (2003): The cognitive basis of translation universals. In: Target 15, 2, S. 197-241.

Halverson, Sandra L. (2015): Cognitive translation studies and the merging of empirical paradigms. The case of ,literal translation‘. In: Translation Spaces 4, 2, S. 310-340.

Krings, Hans P. (1986): Was in den Köpfen von Übersetzern vorgeht. Eine empirische Untersuchung zur Struktur des Übersetzungsprozesses an fortgeschrittenen Französischlernern. (= Tübinger Beiträge zur Linguistik 291). Tübingen.

Levelt, Willem J.M. (1999): Producting spoken language: A blueprint of the speaker. In: Brown, Colin M./Hagoort, Peter (Hg.): The neurocognition of language. New York, S. 83-122.

Marcus, Mitchell P./Marcinkiewicz, Mary A./Santorini, Beatrice (1993): Building a large annotated corpus of English: The Penn Treebank. In: Computational Linguistics 19, 2, S. 313-330.

Nitzke, Jean (2016): Monolingual post-editing. An exploratory study on research behaviour and target text quality. In: Hansen-Schirra, Silvia/Gruzca, Sambor (Hg.): Eyetracking and applied linguistics. (= Translation and Multilingual Natural Language Processing 2 ). Berlin, S. 83-108.

Nitzke, Jean (i.Vorb.): Problem solving activities in post-editing and human translation a multi-method study. Dissertation. Germersheim.

Oster, Katharina (i.Vorb.): Reorganisation of the mental lexicon during translation. Dissertation. Germersheim.

R Core Team (2000): R language definition. Wien.

Schaeffer, Moritz/Carl, Michael (2013): Shared representations and the translation process. A recursive model. In: Translation and Interpreting Studies 8, 2, S. 169-190.

Schaeffer, Moritz et al. (2016): Word translation entropy: Evidence of early target language activation during reading for translation. In: Carl/Bangalore/Schaeffer (Hg.), S. 183-210.

Shannon, Claude E. (1948): A mathematical theory of communication. In: Bell System Technical Journal 27, 3, S. 379-423.

Teich, Elke (2003): Cross-linguistic variation in system and Text. A methodology for the investigation of translations and comparable texts. (= Text, Translation, Computational Processing (TTCP) 5). Berlin.

Tirkkonen-Condit, Sonja (2004): Unique items-over-or under-represented in translated language? In: Mauranen, Anna/Kujamäki, Pekka (Hg.): Translation universals: Do they exist? (= Benjamins Translation Library 48). Amsterdam/Philadelphia, S. 177-186.

Wiersema, Jan R./van der Meere, Jacob J./Roeyers, Herbert (2007): Developmental changes in error monitoring: An event related potential study. In: Neuropsychologia 45, 8, S. 1649-1657.

Wilss, Wolfram (1996): Knowledge and skills in translator behavior. (= Benjamins Translation Library 15). Amsterdam/Philadelphia. 